\title{
Left to right atrial shunting in tricuspid atresia
}

\author{
P SYAMASUNDAR RAO \\ From the Departments of Pediatrics, Medical College of Georgia, Augusta, Georgia, USA
}

SUMMARY In tricuspid atresia, an obligatory right to left shunt occurs at the atrial level. We have observed several patients with left to right interatrial shunts. Data from cardiac catheterisation in 40 consecutive patients were reviewed to determine the frequency and mechanism of left to right shunting in triscuspid atresia. An increase of $6 \%$ or more in oxygen saturation between the superior vena cava and the right atrium in two or more sets of saturations, representing a left to right shunt, was present in 29 out of $50(58 \%)$ catheterisations in which the data were adequate. In most, the shunt was also seen cineangiographically in the laevophase.

In only two catheterisations was an anatomical cause (ostium primum atrial septal defect in one and anomalous pulmonary venous return in the other) found. In the remaining 27 catheterisations, no anatomical cause was found. Age, Qp:Qs, and mean atrial pressure difference were similar between the shunt and non-shunt groups. In the shunt group right atrial "a" waves were equal to or higher than left atrial "a" waves and left atrial "v" waves were equal to or higher than right atrial "v" waves. Simultaneous pressure recordings (in one patient with left to right atrial shunt) from the left atrium and right atrium with isosensitised miniature pressure transducers mounted $5 \mathrm{~cm}$ apart showed (1) a higher pressure in the right atrium than in the left atrium during atrial systole and (2) a higher pressure in the left atrium than in the right atrium during atrial diastole. It is concluded that (a) left to right shunt across the atrial septum occurs frequently in tricuspid atresia and (b) the left to right shunt is the result of instantaneous pressure differences between the atria.

In tricuspid atresia, by necessity, right to left shunting occurs at the atrial level. Catheterisation data reported by several authors ${ }^{1-4}$ have shown evidence for left to right shunting, but this has been ignored; Fergusson et al. ${ }^{5}$ however, did comment on the left to right shunt observed in five of the 12 patients in his group who were catheterised. We also observed several patients with tricuspid atresia with left to right atrial shunts. The purpose of this study is to determine if this is a frequent occurrence and, if so, what was the mechanism.

\section{Subjects and methods}

Cardiac catheterisation and angiographic data of 40 consecutive patients with tricuspid atresia seen over a 16 year period (1965-80) at the Medical College of Georgia were reviewed. Thirty-seven of these 40 patients had catheterisation on 67 occasions. Cardiac catheterisation was performed after premedication with a mixture of meperidine $(2 \mathrm{mg} / \mathrm{kg}$ body weight,

Present address: King Faisal Specialist Hospital, Riyadh, Saudi Arabia. Accepted for publication 23 December 1982 maximum $50 \mathrm{mg})$, chlorpromazine $(0.6 \mathrm{mg} / \mathrm{kg}$, maximum $15 \mathrm{mg})$, and promethazine $(0.6 \mathrm{mg} / \mathrm{kg}$, maximum $15 \mathrm{mg}$ ) in patients older than 2 months. Younger patients did not receive any premedication. An increase of $6 \%$ or more in oxygen saturation between the superior vena cava and the right atrium mid-lateral position in two or more sets of saturations ${ }^{6}$ was considered to represent a left to right shunt. Adequate data to evaluate the interatrial left to right shunting were present in 50 catheterisations. The age at catheterisation varied between 1 day and 19 years, with a median of 1.5 years and a mean of 4.1 years. Phasic and mean pressures recorded with fluid filled catheters, usually obtained on a pullback from the left atrium to the right atrium, cineangiograms recorded during the catheterisation, and diagnoses with particular attention to associated defects causing left to right shunts at atrial level were reviewed. Pulmonary to systemic flow ratio ( $Q p: Q s)$ was calculated using the formula. ${ }^{6}$

$$
\mathrm{Qp}: \mathrm{Qs}=\frac{\text { Ao }(\text { FA }) \text { Sat-SVC Sat }}{\text { PV Sat-PA Sat }}
$$

where $A o=$ aorta, $F A=$ femoral artery, $P A=$ pul- 
monary artery, $\mathrm{PV}=$ pulmonary vein, Sat $=$ oxygen saturation \%, and $S V C=$ superior vena cava.

In patients in whom aortic or femoral artery oxygen saturations were not obtained, the left ventricular oxygen saturations were used instead. Similarly, left ventricular saturation was used if pulmonary arterial saturation could not be obtained. If the pulmonary vein was not entered $96 \%$ oxygen saturation was assumed.

In a 2 month old infant with tricuspid atresia without transposition, but with left to right atrial shunt documented by oximetry and by angiography, simultaneous pressure recordings from the left atrium and right atrium were obtained with two isosensitised miniature pressure transducers (Millar, Mikro-tip Pressure Transducer, PC-761) mounted $5 \mathrm{~cm}$ apart (Fig. 1). The statistical significances were determined by using Student's t test where appropriate.

\section{Results}

In 29 of the 50 catheterisations there was evidence for left to right interatrial shunting (Fig. 2). The mean increase in oxygen saturation between the superior vena cava and the right atrium was $11.8 \%(S D \pm 5)$, with a range of 6 to $23 \%$. Left to right atrial shunting was confirmed on the "laevo" phase of the cineangiograms in 19 studies (Fig. 3). In the remaining 10 studies, the "laevo" phase was too faint to evaluate the shunting. Among the 21 patients without interat- $c$ rial left to right shunts on oximetry, there was no $\vec{F}$ angiographic "laevo" phase evidence for left to right atrial shunting in 15 , and in the remaining six, the "laevo" phase was too faint to give this information.

In only two patients was an anatomical cause for left to right atrial shunt present; in one there was a large ostium primum atrial septal defect and in the other, total anomalous pulmonary venous connection. In the $\vec{\circ}$ remaining 27 catheterisations, no anatomical cause was found. The two catheterisations with anatomical cause were excluded from further analysis. The left to right shunting was present in both type I (normally related great arteries) and type II (transposition) ${ }^{7}$ patients; $54 \%$ (19 out of 35 ) of catheterisations in type I patients and $62 \%(8 / 13)$ in type II patients had left to right shunts.

The age at catheterisation in the shunt group varied from 2 days to 18 years, with a median of 2.5 years and a mean of 3.4 years $(S D \pm 4.7)$. In the group without the shunt, the age range was 1 day to 19 years, median was 2.5 years and the mean was 4.1 years $(\mathrm{SD} \pm 5 \cdot 2)$. There was no statistical difference between these two groups $(p>0 \cdot 1)$.

The pulmonary to systemic flow ratios are listed in the Table. Though the group with an atrial shunt had a wider range of $Q p: Q s$ and larger mean values, these differences are not statistically different $(p>0 \cdot 1)$. It

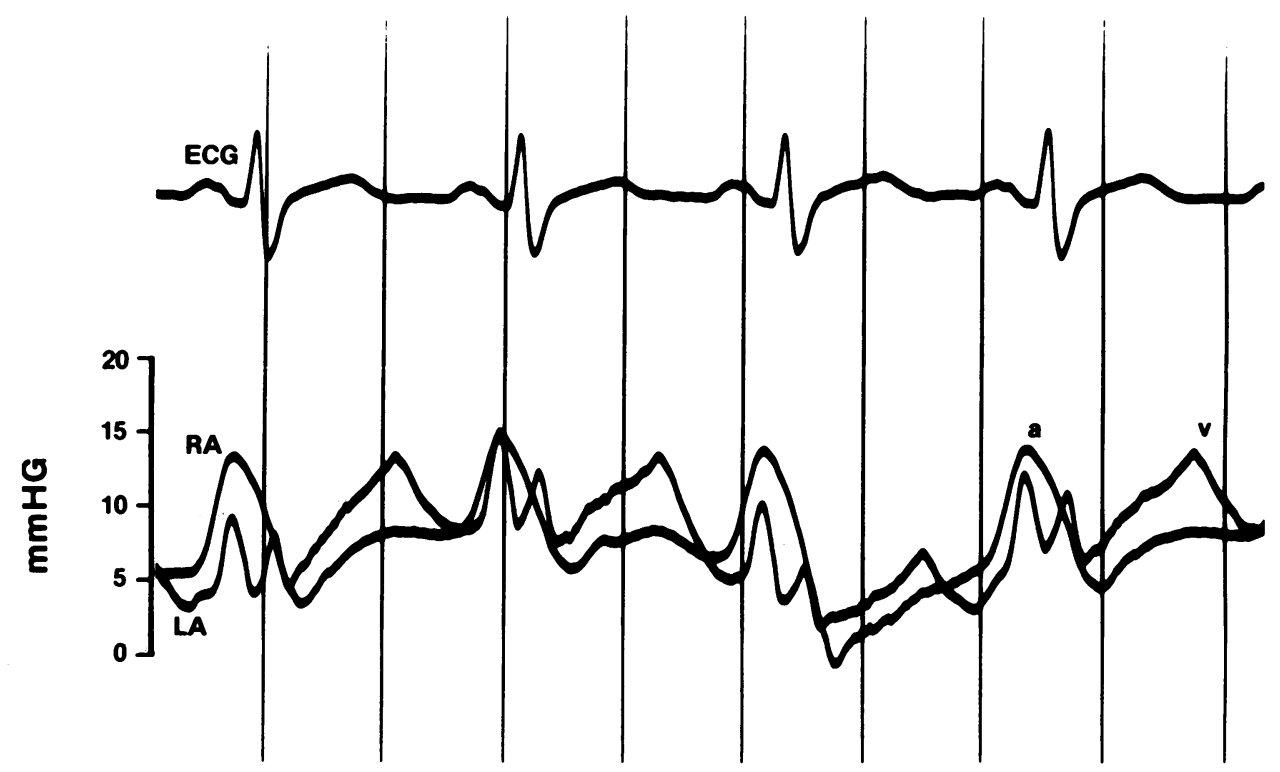

Fig. 1 Simultaneous pressure tracings from the left atrium and right atrium recorded by means of high fidelity miniature transducers mounted on a catheter $5 \mathrm{~cm}$ apart. Note higher right atrial pressure during atrial systole and higher left atrial pressure during atrial diastole. $a$, " $a$ " wave; ECG, electrocardiogram; $v$, "v" wave. 


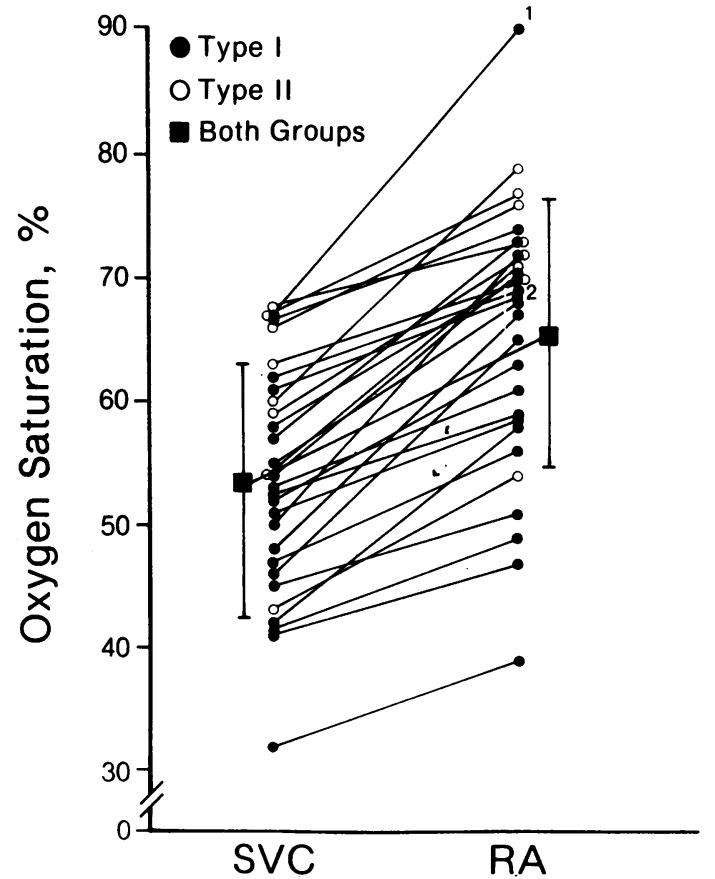

Fig. 2 Oxygen saturation (\%) in the superior vena cava (SVC) and right atrium $(R A)$ in 29 catheterisations. Closed circles are from patients with type I (normally related great arteries) and open circles from type II (transposition of the great arteries) cases of tricuspid atresia. The mean and one standard deviation of the data from the SVC and RA are marked; there is a significant increase in oxygen saturation ( $p<0.001)$. Oxygen saturation data marked 1 are from a patient with anomalous pulmonary venous retum and 2 are from a patient with a large ostium primum atrial septal defect. The increases in oxygen saturation from the SVC to $R A$ in type I vs type $I I$ patients are not statistically different $(p>0.1)$ (not shown in Fig).

should also be noted that left to right shunt occurred in patients with conspicuously decreased Qp:Qs, namely 0.4 , whereas no shunt was demonstrated in some patients with high $\mathrm{Qp}: \mathrm{Qs}$, for example, 5.0, thus suggesting that the magnitude of $Q p: Q s$ does not determine left to right interatrial shunt in tricuspid atresia.
The mean pressure in the right atrium was higher than that in the left atrium in both groups (Table), but yet, left to right atrial shunts occurred in the group with an atrial shunt. The mean pressure differences were not statistically different $(p>0 \cdot 1)$ between groups (Table). Thus, the mean pressures in the atria do not appear to influence the presence or absence of left to right atrial shunts in tricuspid atresia.

In order to determine the mechanism of left to right atrial shunting in tricuspid atresia, the phasic pressures in both atria were anaiysed. In the group without shunt, the right atrial " $a$ " wave was equal to or higher than the left atrial "a" wave in 16 of the 18 catheterisations in which the data were available. In the remaining two, the right atrial " $a$ " wave was lower than that in the left atrium. The left atrial " $v$ " wave was equal to or lower than that in the right atrium in 10 catheterisations. In the remaining eight, the left atrial "v" wave was higher, but in six of these the pressure difference was less than $1 \mathrm{mmHg}$, and in only two was the pressure difference greater than $2 \mathrm{mmHg}$. In the shunt group, the "a" wave in the right atrium was equal to or higher than that in the left atrium in 23 of the 26 catheterisations in which the data were available. In the remaining three, the right atrial " $a$ " wave was lower than that in the left atrium. The left atrial " $v$ " wave was equal to or higher than that in the right atrium in 24 catheterisations and lower in the remaining four. Therefore, it appears that the interatrial right to left shunting occurs during atrial systole during which there was an atrial pressure gradient in favour of the right atrium and left to right atrial shunting occurs during atrial diastole during which period the pressure gradient favoured the left atrium. These pressures, however, were measured from a pullback tracing from the left to the right atrium and not recorded simultaneously. Simultaneous biatrial manometry may be valuable in showing the phasic nature of the bidirectional shunting, as has been shown in transposition of the great arteries. ${ }^{8}$ Therefore, we recorded pressures simultaneously from both atria with isosensitised catheter mounted miniature pressure transducers (Fig. 1). The pressure in the right atrium exceeded that in the left atrium

Table Pulmonary to systemic flow ratios and mean right atrial to left atrial pressures in both groups

\begin{tabular}{llllll}
\hline & No. & Range & Mean & $S D$ & $p$ value \\
\hline Qp:Qs shunt group & 27 & 0.4 to 6.4 & 2.5 & \pm 1.5 & $>0.1$ \\
No shunt group & 21 & 0.1 to 5.0 & 1.8 & \pm 1.4 & \\
$\begin{array}{l}\text { Mean RA-LA pressure (mm Hg) } \\
\text { shunt group }\end{array}$ & 27 & -1 to +3 & +0.8 & \pm 1.1 & $>0.1$ \\
No shunt group & 19 & -1 to +3 & +1.2 & \pm 1.2 & \\
\hline
\end{tabular}

LA, left atrium; No., number of catheterisations; Qp:Qs, pulmonary to systemic flow ratio; RA, right atrium; SD, standard deviation. 

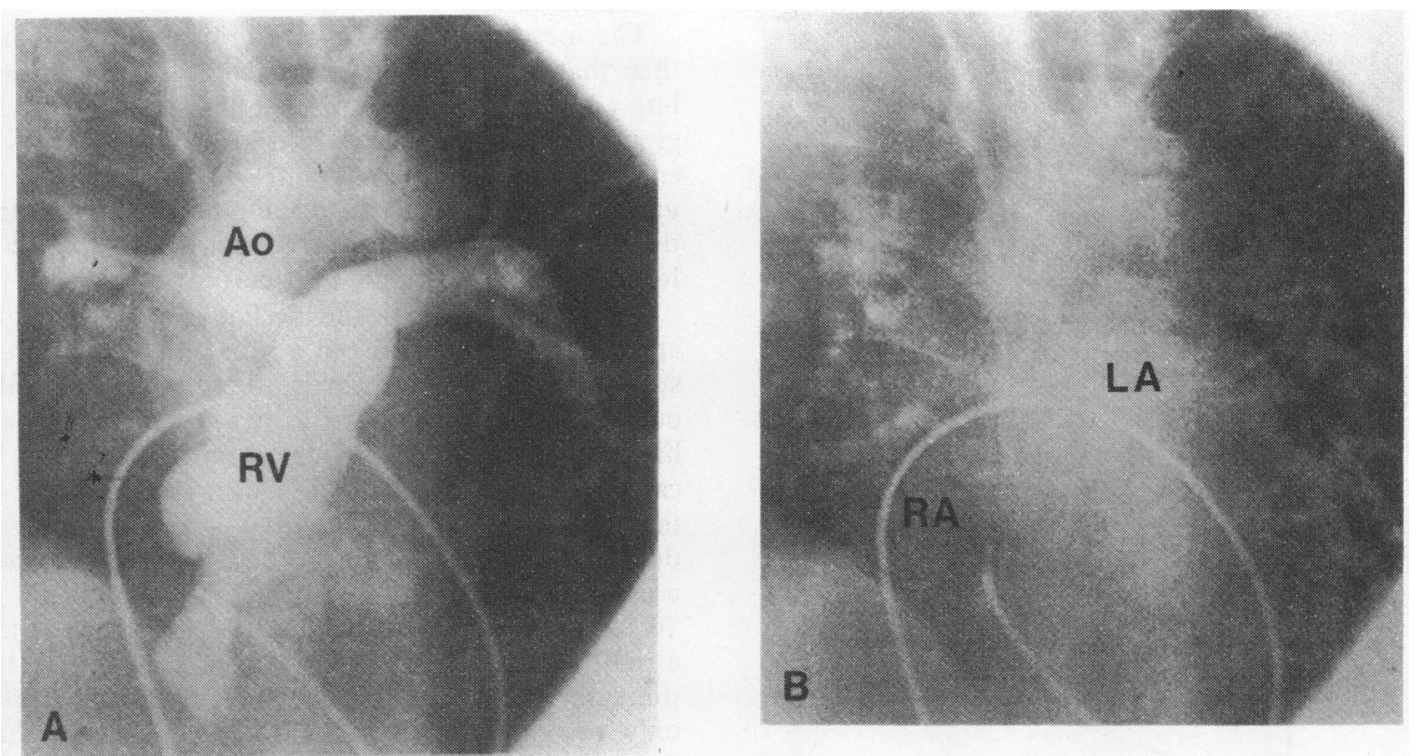

Fig. 3(A) A selected frame from the right ventricular cineangiogram obtained in a four chamber view (45 lefi anterior oblique with $35^{\circ}$ cranial angulation). The catheter has been advanced from the right atrium into the left atrium, into the left ventricle and from there into the right ventricle via a ventricular septal defect. Severe stenosis in the mid-right ventricle $(R V)$ and opacification of the pulmonary arteries are noted. $(B)$ On the laevophase of the above cineangiogram, the left atrium (LA) and left ventricle were opacified. The right atrium $(R A)$ was opacified via the patent foramen ovale. On the cine, a definite jet of contrast material was seen from the left atrium to the right atrium ( $L A$ to $R A$ ). This child had an increase of $8 \%$ oxygen saturation from the superior vena cava to the right atrium Ao, aorta.

during atrial systole and pressure in the left atrium was higher than that in the right atrium during most of atrial diastole.

\section{Discussion}

Because of atresia of the tricuspid valve, obligatory right to left atrial shunting occurs, usually through a patent foramen ovale. Because of this obligatory shunting it is surprising to see left to right interatrial shunting. Since this is unexplained, the finding has been apparently ignored by many previous investigators. ${ }^{1-4}$ Fergusson et al. ${ }^{5}$ suggested that this shunt might be occurring in some phase of the cardiac or respiratory cycles. It was our impression that left to right atrial shunt occurs occasionally in tricuspid atresia, but we were surprised to find a very high incidence, $56 \%$ (27/48), even after exclusion of two anatomical causes of the shunt.

The mixing of the inferior and superior vena caval and coronary sinus blood streams is inadequate in the right atrium and different oxygen saturations may be measured at different sites in the atrium. ${ }^{6}$ The midlateral position in the right atrium is considered to have the most consistent values ${ }^{6}$ and is the routine location for sampling from the right atrium in our laboratory. In spite of this, the increase in the right atrial oxygen saturation may be secondary to high renal venous oxygen saturation (streaming). Especially smaller ranges of step-up (6 to 10\%) (Fig. 2) could be the result of this. The higher levels of oxygen step-ups (11\% to $23 \%$ ), however, could not be explained by the high renal vein saturations. Furthermore, on the laevo cineangiographic phase we observed left to right shunt across the atrial septum in most of these patients and, therefore, the left to right shunt that we report is real.

Once the left to right atrial shunt was documented, we sought to identify the causes and mechanisms of the shunt. An anatomical cause was found in two $\mathrm{N}$ children (one with large ostium primum atrial septal defect and another with total anomalous pulmonary 0 venous connection). In the remaining 27 catheterisations, the shunting was "physiological". Age did not seem to have any effect; both young and older chil- $\bullet$ dren showed shunting. Large pulmonary flows are $\mathbb{\Phi}$ likely to produce enlargement of the left atrium and stretching of the patent foramen ovale which in turn might predispose to left to right atrial shunting; it $\frac{\pi}{\pi}$ occurred in patients with a small and very decreased Qp:Qs as well as in those with a large Qp:Qs. Furth- $\stackrel{\mathbb{Q}}{\varrho}$ ermore, the Qp:Qs in the group with a shunt was

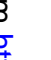


similar to that in the group without one $(p>0 \cdot 1)$. Similarly, the mean pressure differences between atria were also similar $(p>0.1)$ in both groups; the right atrial pressure was higher than that in the left atrium in most patients of both groups. Thus, the magnitude of Qp:Qs or atrial mean pressure differences did not seem to be related to atrial left to right shunt.

Analysis of the phasic pressure differences in the shunt group suggested a pressure gradient in favour of the right atrium during atrial systole (" $a$ " wave) and in favour of the left atrium during atrial diastole (" $v$ " wave). Simultaneous pressure tracings from both atria in one patient with left to right shunt confirmed these observations and suggested that there were instantaneous pressure differences between atria (Fig. 1), presumably responsible for left to right atrial shunts in tricuspid atresia.

Levin and associates 9 studied the dynamics of interatrial right to left shunting in nine patients with tricuspid atresia by simultaneous pressure recordings from both the atria with fluid filled catheters and by biplane cineangiography. They found that the right to left shunt across the interatrial septum occurred during the right atrial systole and showed a pressure gradient in favour of the right atrium. Our data are in agreement with theirs in that a higher pressure in the right atrium than that in the left atrium was observed during atrial systole. These workers, however, did not detect any consistent pressure gradient in favour of the left atrium during atrial diastole nor did they report oxygen saturation data to suggest left to right shunt at atrial level in their nine patients with tricuspid atresia, but they observed small left to right shunts across the foramen ovale during atrial diastole (ventricular systole) after pulmonary vein cineangiograms, and commented that this interatrial left to right shunting was difficult to interpret because the catheter was positioned across the interatrial communication. Had they looked at the laevoangiographic phase of the angiogram and carefully obtained oxygen saturations from the superior vena cava and right atrium, they might have detected the left to right shunt in some case of tricuspid atresia, as we did.

\section{CLINICAL IMPLICATIONS}

In tricuspid atresia when a higher oxygen saturation in the right atrial mid-lateral position than in the superior vena cava is detected, it is important to look for anatomical causes, such as a large atrial septal defect, anomalous pulmonary venous connection, coronary arteriovenous fistula to the right atrium, sinus of Valsalva fistula to the right atrium, or other even rarer causes. Based on our experience, anatomical causes of atrial left to right shunting in tricuspid atresia are rare. Once the anatomical cause is excluded, the shunting is physiological in nature and is caused by instantaneous pressure differences between atria and is of no concern.

The author wishes to acknowledge the valuable contribution of Drs Bruce Alpert, Wesley Covitz, and William Strong in the preparation of the manuscript. Rollie Harp, M.S. of the Systems and Computer Services of the Medical College of Georgia, Augusta, assisted in the statistical analysis.

\section{References}

1 Gallaher ME, Fyler DC. Observations on changing hemodynamics in tricuspid atresia without associated transposition of the great vessels. Circulation 1967; 35: 381-8.

2 Marcano BA, Riemenschneider TA, Ruttenberg HD, Goldberg SJ, Gyepes M. Tricuspid atresia with increased pulmonary blood flow: an analysis of 13 cases. Circulation 1969; 40: 399-410.

3 Shaher RA, Farina M, Kausel H. Spontaneous closure of ventricular septal defect: tricuspid atresia following Waterston's anastomosis. New York State f Med 1973; 73: 455-8.

4 Rao PS. Natural history of the ventricular septal defect in tricuspid atresia and its surgical implications. $\mathrm{Br} \mathrm{Heart} \mathrm{f}$ 1977; 39: 276-88.

5 Fergusson DJG, Schrire V, Vogelpoel L, Beck W. Tricuspid atresia. S Afr Med F 1964; 38: 864-7.

6 Rudolph AM. Congenital diseases of the heart. Chicago: Year Book Medical Publishers, 1974: 49-165.

7 Rao, PS. A unified classification for tricuspid atresia. Am Heart .7 1980; 99: 799-804.

8 Carr I. Timing of bidirectional atrial shunts in transportation of the great arteries (TGA) and atrial septal defect (ASD) [Abstract]. Circulation 1971; 44 (suppl II): 11-70.

9 Levin AR, Spach MS, Canent RV Jr, Boineau JP. Dynamics of interatrial shunting in children with obstruction of the tricuspid and pulmonic valves. Circulation 1970; 41: 503-12.

Requests for reprints to Dr P Syamasundar Rao, Department of Pediatrics, King Faisal Specialist Hospital, PO Box 3354, Riyadh, Saudi Arabia. 